discussion of a systematic approach to disease nomenclature that incorporates the clinical or phenotypic diagnosis and the relevant etiologic factors, be they genetic or environmental. In the editorial, J.M. Friedman ${ }^{2}$ suggests that the ACMG might develop an appropriate system.

In 1991, at the 8th ICHG meeting in Washington, I first proposed the use of a multiaxial diagnostic system (MADS) in clinical genetics ${ }^{3}$ similar to the DSM-III-R system used in psychiatry. I thought this was important as the clinical diagnoses, and even the ICD codes, were a mixture of etiologic, pathogenetic mechanisms and phenotypic diagnoses. For simplicity, only four major categorical axes (I, Phenotypic; II, Pathogenetic; III, Etiologic, and IV, Differential Diagnoses) were defined. The major advantages of such a system included improved diagnostic accuracy; greater reliability and homogeneity of the diagnostic categories, enhancing the comparison of diagnoses and achievements between different centers, states, regions, or countries; more comprehensive and specific definitions for controversial terms such as "syndrome" or "spectrum"; and its use for educating health professionals.

I recognized that the system would need periodic revisions to keep it consistent and conceptually accurate, in accordance with advances in genetics. A basic description was published in $1994^{4}$ after its presentation at the International Birth Defects Meeting organized by Bartsocas in Greece. In that meeting we pointed out how it could easily be updated in the future in accordance with the new molecular or biochemical information. Further updates were presented at the subsequent ICHG meetings in Brazil (1996) and recently in Austria (2001). In Vienna I stressed its usefulness for increasing the percentage of etiologic diagnoses established. ${ }^{5}$ In several other publications $^{6-9}$ I have mentioned or further described this system. Therefore, after more than 12 years of using it, I couldn't be more in agreement with Robin and Biesecker and Friedman regarding the importance of using this kind of system in clinical genetics.

The principal goal of a clinical geneticist is to establish not only the phenotype and pathogenetic mechanism, if known, but to determine the etiologic diagnosis, which is the cornerstone for genetic counseling. This is Axis III in our MADS. In this axis we consider not only the classical Mendelian, chromosomal, polygenic/multifactorial, and environmental (teratogens) etiologies, but also the new, nontraditional etiologies or mechanisms that have been described in the past 10 to 20 years (contiguous gene syndromes or microdeletions, mitochondrial mutations, uniparental disomy, mosaicism, triple repeat expansions, etc.). Still new ones may yet be described.

There is no question that molecular information should be incorporated into the MADS. We all agree that syndromes that we thought were similar may have completely different gene mutations, and syndromes we suspected were different conditions have been shown to have sometimes similar mutations, or be allelic. The system I proposed allows the incorporation of this new information. I think it is a good idea to include modifiers of Axis I (the phenotype) such as age of onset and to

\section{Use of a multiaxial diagnostic system in clinical genetics}

\section{To the Editor:}

In the July/August 2001 issue of Genetics in Medicine, Robin and Biesecker ${ }^{1}$ suggest a multiaxis nomenclature system for medical genetics. The purpose of their proposal is to initiate systematically include the OMIM numbers for Mendelian dis- 
orders in Axis III as Robin and Biesecker suggest. In Brazil and Austria, I also insisted on the convenience of incorporating this kind of nomenclature into the ICD system. This idea was immediately envisioned by the late Dr. Frank Greenberg when he learned about the MADS. He asked me for further information in order to include our system in the ICD, as Robin and Biesecker suggest.

Although I don't agree with the dichotomy that Robin and Biesecker present of phenotypic versus etiologic and their proposed axes, I think further discussion and adoption of an international system will certainly be an important contribution to the field of clinical genetics.

Yves Lacassie, MD, FACMG

Division of Clinical Genetics

Department of Pediatrics

Louisiana State University Health Sciences Center and Children's

Hospital of New Orleans, Louisiana

\section{References}

1. Robin NH, Biesecker LG. Considerations for a multiaxis nomenclature system for medical genetics. Genet Med 2001;3:290-293.

2. Friedman JM. Do geneticists need Babel fish? Genet Med 2001;3:283-284.

3. Lacassie Y. Use of a multiaxial diagnostic system in clinical genetics. Am J Hum Genet 1991;suppl 49(4):146.

4. Lacassie Y. An international multiaxial diagnostic system in clinical genetics. In: Bartsocas CS, Beighton P, editors. Dysmorphology and genetics of cardiovascular disorders. Athens: Zerbinis, 1994:28-31.

5. Lacassie Y, LaMotta I, Florez L. Nosology of genetic disorders. Eur J Hum Genet 2001;suppl 1, 9:302(A).

6. Lacassie Y, Arriaza M. Clinical approach to diagnosing craniofacial anomalies: oral and maxillofacial OMS knowledge update, self-study program, Vol 2, pp 19-29. Piecuch J, Editor-in-Chief. American Association of Oral and Maxillofacial Surgeons, May 1998.

7. Lacassie Y. Approach to clinical diagnosis of genetic disorders. Biomedicina 1998;1: 9-16.

8. Lacassie Y. Approach to clinical diagnosis of genetic disorders. Pediatr Rev 1998; $\mathrm{XII}(4): 1-3$.

9. Lacassie Y, LaMotta I, Florez L. Classification and distribution of genetic disorders: the LSU New Orleans/Children's Hospital experience (1986-2000). Annual Clinical Genetics Meeting. Miami, March 1-4, 2001. 